\title{
Environmental Stability and Residual Stresses in Zirconia Femoral Head for Total Hip Arthroplasty: In Vitro Aging versus Retrieval Studies
}

\author{
Masanori Arita, ${ }^{1}$ Yasuhito Takahashi, ${ }^{1,2}$ Giuseppe Pezzotti, ${ }^{3}$ Takaaki Shishido, \\ Toshinori Masaoka, ${ }^{1}$ Keiji Sano, ${ }^{1}$ and Kengo Yamamoto ${ }^{1}$ \\ ${ }^{1}$ Department of Orthopaedic Surgery, Tokyo Medical University, 6-7-1 Nishishinjuku, Shinjuku-ku, Tokyo 160-0023, Japan \\ ${ }^{2}$ Department of Bone and Joint Biomaterial Research, Tokyo Medical University, 6-7-1 Nishishinjuku, Shinjuku-ku, \\ Tokyo 160-0023, Japan \\ ${ }^{3}$ Ceramic Physics Laboratory, Kyoto Institute of Technology, Sakyo-ku, Matsugasaki, Kyoto 606-8585, Japan
}

Correspondence should be addressed to Yasuhito Takahashi; yasuhito@tokyo-med.ac.jp

Received 17 April 2015; Accepted 24 May 2015

Academic Editor: Radovan Zdero

Copyright (C) 2015 Masanori Arita et al. This is an open access article distributed under the Creative Commons Attribution License, which permits unrestricted use, distribution, and reproduction in any medium, provided the original work is properly cited.

\begin{abstract}
The objective of this study was to compare the low temperature degradation (LTD) behavior of femoral heads made of $3 \mathrm{Y}$-TZP as observed on retrievals with that induced in vitro upon prolonged exposures to a hydrothermal environment. The time-dependent evolution of tetragonal-to-monoclinic transformation and the related residual stresses were nondestructively monitored by Raman microspectroscopy. An increasing intensification of tensile and compressive stresses was detected with increasing hydrothermal aging duration in tetragonal and monoclinic phases, respectively. The dependence of monoclinic fraction upon exposure time was rationalized through the Mehl-Avrami-Johnson (MAJ) formalism in order to interpret the LTD process according to a two-step mechanism of formation and growth of monoclinic nuclei. In vitro results were compared to in vivo monoclinic contents in the same type of 3Y-TZP head retrievals after implantation periods of 1.6-16.6 y, also including literature data previously reported by other authors. One-hour exposure under the selected aging condition is estimated to correspond to in vivo exposures of 4 and 2 years according to ISO and ASTM criteria, respectively. A critical review of these two criteria according to the present analyses revealed that the ASTM simulation predicts more closely the in vivo results as compared to the ISO one.
\end{abstract}

\section{Introduction}

Zirconia $\left(\mathrm{ZrO}_{2}\right)$ bearings have been considered as a valid alternative to alumina bearings in total joint replacement (TJR). The most attractive characteristics of $\mathrm{ZrO}_{2}$ are its excellent flexural strength and fracture toughness, which are significantly higher than those of alumina $\left(\mathrm{Al}_{2} \mathrm{O}_{3}\right)[1]$. So far, the $\mathrm{ZrO}_{2}$ material used in orthopedics has predominantly consisted of a partially stabilized tetragonal phase with a content of $3 \mathrm{~mol} \%$ of yttria $\left(\mathrm{Y}_{2} \mathrm{O}_{3}\right)$, which is typically referred to as $3 \mathrm{Y}$-TZP (i.e., $3 \mathrm{~mol} \% \mathrm{Y}_{2} \mathrm{O}_{3}$-stabilized tetragonal $\mathrm{ZrO}_{2}$ polycrystal) [1-3]. Unlike metallic joint implants made of cobalt-chrome or stainless steel [4], oxide ceramics are supposed to be more stable in biological environment since, in principle, they possess less driving force for structural degradation in air. Nevertheless, it has been well recognized that, in human body, the 3Y-TZP material is metastable and can transform from the tetragonal to the monoclinic $(t \rightarrow m)$ polymorph under the combined effects of biological (aqueous) and mechanical stress environments (e.g., frictional wear, body weight, and impingement). When acting against advancing cracks, this phenomenon plays a significant role in increasing fracture toughness by generating a strong crackshielding effect as a consequence of $3 \sim 4 \%$ volume expansion (i.e., a mechanism referred to as transformation toughening) $[1-3,5]$. However, if the $t \rightarrow m$ transformation becomes environmentally driven and uncontrollably occurs prior to crack propagation, the material undergoes a significant loss of 
TABLE 1: Summary of the clinical characteristics of the 3Y-TZP femoral heads investigated in this study.

\begin{tabular}{|c|c|c|c|c|c|}
\hline Femoral head sample & Diameter & Maker & State & Follow-up & $\begin{array}{l}\text { Cause of } \\
\text { revision }\end{array}$ \\
\hline Head (I) & $28 \mathrm{~mm}$ & $\begin{array}{l}\text { Saint-Gobain } \\
\text { Desmarquest }\end{array}$ & $\begin{array}{c}\text { Unused or } \\
\text { in-vitro aged at } \\
134^{\circ} \mathrm{C}\end{array}$ & - & - \\
\hline Head (II) & $28 \mathrm{~mm}$ & $\begin{array}{c}\text { Saint-Gobain } \\
\text { Desmarquest }\end{array}$ & Retrieval & $15.1 \mathrm{yrs}$ & Infection \\
\hline Head (III) & $28 \mathrm{~mm}$ & $\begin{array}{c}\text { Saint-Gobain } \\
\text { Desmarquest }\end{array}$ & Retrieval & $16.6 \mathrm{yrs}$ & $\begin{array}{l}\text { UHMWPE liner } \\
\text { wear }\end{array}$ \\
\hline
\end{tabular}

crack growth resistance, strength, and reliability [1-3,5]. This latter mechanism is usually referred to as low temperature degradation (LTD) and has been regarded as the "Achilles' heel" of 3Y-TZP when employed in load-bearing applications, leading to catastrophic fracture $[1,2,6]$ and surface roughening $[2,3,7]$. Since the above two mechanisms occur competitively during in vivo implantation, the time-dependent LTD phenomenon makes orthopedic surgeons hesitant about using $\mathrm{ZrO}_{2}$ at the time of arthroplastic intervention.

In the above contexts, the stability of the tetragonal phase can largely affect the clinical outcome and long-term survivorship of joint implants made of 3Y-TZP prosthesis. Thus, it is of great importance to experimentally simulate the time-dependence of LTD behavior of 3Y-TZP and the related changes in residual stress fields at its bearing surface, which could provide a reasonable prediction of in-vivo performance and durability. The aim of this study is to estimate to which extent an in vitro aging test, standardized for biomedical grade $\mathrm{ZrO}_{2}$, could be valid for predicting the in vivo resistance to LTD. For this purpose, we artificially accelerated the LTD phenomenon in commercially available 3Y-TZP femoral heads by means of a prolonged exposure to water vapor atmosphere $\left(134^{\circ} \mathrm{C}, 2\right.$ bars of pressure) on the basis of ISO and ASTM testing conditions [8-11]. The time-dependent degradation of 3Y-TZP heads was assessed by means of confocal Raman microprobe spectroscopy in terms of increases in monoclinic volume fraction as well as in surface residual stress magnitude. In order to screen nondestructively the aging behaviors, the spectroscopic evaluations were made in confocal mode from the articular surface down to subsurface regions of the femoral heads. Furthermore, the LTD kinetics of the in vitro aged heads was compared with that obtained from head retrievals of the same brand subjected to long-term implantation in vivo ( $>15$ years). Comparisons were also made with the surface monoclinic fractions analyzed previously by other authors for short- and middle-term retrievals (1.6 11 years) via X-ray diffraction (XRD) analysis [12-14].

\section{Materials and Methods}

2.1. Unused 3Y-TZP Femoral Heads and In Vitro Aging Test. The $28 \mathrm{~mm}$ sized 3Y-TZP femoral heads $(n=3)$ were provided by Biomet Inc. (Warsaw, IN). These were manufactured by Saint-Gobain Desmarquest Inc. (Evreux, France). These heads were distributed with the trade name of Prozyr (currently out of market since 2001). All the Prozyr heads used in this study were produced by batch-furnace sintering, and not by tunnel furnace sintering which historically had resulted in the unprecedented number of reports of fractured Prozyr heads $[2,6]$. The head samples were subjected to no further washing or manipulation prior to in vitro aging test and spectroscopic characterizations. The average grain size of the heads was $0.5 \mu \mathrm{m}$. In this paper, the as-received samples are simply referred to as "Head (I)" (Table 1). The accelerated aging test was performed at $134^{\circ} \mathrm{C}$ under 2 bars water steam, namely, the conditions that satisfy both ISO and ASTM standards [8-11]. The aging test was carried out at $2.5 \mathrm{~h}$ intervals up to $20 \mathrm{~h}$ using a high-pressure steam sterilizer (TOMY SX-300, Tomy Seiko, Co., Tokyo, Japan). After sequential intervals of $2.5 \mathrm{~h}$ in autoclave, the monoclinic content and the residual stress field were monitored using confocal Raman microprobe spectroscopy (cf. forthcoming Section 2.3).

2.2. Retrievals of 3Y-TZP Femoral Heads and Their Clinical Background. In order to compare the in vitro LTD behavior of the pristine Head (I) with the actual in vivo performance of the same material, two Prozyr femoral head retrievals were also investigated by Raman microprobe spectroscopy. These retrievals were implanted in female patients for 15.1 and 16.6 years (i.e., henceforth referred to as Heads (II) and (III), 
resp.). Clinical information is summarized for both heads in Table 1. The diameter of Heads (II) and (III) was $28 \mathrm{~mm}$. Both heads articulated against acetabular liners made of conventional (noncrosslinked) ultra-high molecular weight polyethylene (ArCom, Biomet Inc., Warsaw, IN) with the outer diameter of $48 \mathrm{~mm}$. The causes of revision surgery were infection (Head (II)) and liner wear (Head (III)), respectively. It should be noted that both the retrieval heads were implanted without significant positioning error at the time of primary surgery, as far as the cup and stem orientation were concerned.

2.3. Raman Microprobe Spectroscopic Analyses. The monoclinic volume fraction, $V_{m}$, and the residual (tensor trace) stresses, $\sigma$, of Head (I) (III) were quantitatively measured by means of a Raman microprobe spectrometer (MS3504i, SOL instruments Ltd., Minsk, Republic of Belarus). The excitation source was a $488 \mathrm{~nm}$ Ar-ion laser (GLG3103, Showa Optronics Co., Ltd., Tokyo, Japan) yielding a power of approximately $35 \mathrm{~mW}$ on the sample surfaces. The confocal configuration of the probe adopted throughout the present experiments corresponded to a $\times 100$ objective; numerical aperture, focal length, and pinhole diameter were fixed as $0.6,7.6 \mathrm{~mm}$, and $100 \mu \mathrm{m}$, respectively. Individual spectra were typically collected in 5 seconds. All the spectra were acquired in backscattering geometry with a spectral resolution of $\sim 1.5 \mathrm{~cm}^{-1}$ achieved by a 2400 grooves $/ \mathrm{mm}$ grating. The recorded spectra of three successive measurements were averaged. The focal plane was eventually shifted from the articular surfaces down to $100 \mu \mathrm{m}$ insides in order to screen nondestructively the subsurface regions of the femoral heads. An in plane sampling of $2.5 \mu \mathrm{m}$ lateral steps was applied at each depth and a spectral map of $50 \times 50 \mu \mathrm{m}^{2}$ dimensions was collected (for a total of 1323 spectra per each map). A total of 2205 different locations ( $=441$ points per map $\times 5$ maps) were analyzed at each selected depth of the femoral heads.

Spectral deconvolution of all the recorded spectra into subbands was performed according to a mixed Gaussian/Lorentzian curve fit using computational software (Labspec 3, HORIBA Jobin-Yvon SAS, Lille, France). The band intensities and peak positions were calculated after spectral fitting, and the computations of $V_{m}$ and $\sigma$ were made according to (1) and (2), respectively [15-17]:

$$
\begin{aligned}
V_{m} & =\frac{0.5\left(I_{m}^{180}+I_{m}^{190}\right)}{2.2 I_{t}^{150}+0.5\left(I_{m}^{180}+I_{m}^{190}\right)}, \\
\sigma & =\frac{\Delta \nu}{\Pi},
\end{aligned}
$$

where $I_{t}$ and $I_{m}$ represent the intensities of the Raman bands from tetragonal and monoclinic phases in the $3 \mathrm{Y}-$ TZP, whose wavenumbers are identified by the subscript. $\Pi$ and $\Delta v$ represent the piezo-spectroscopic (PS) coefficient and the spectral shift of a Raman peak. Since $\Pi$ values were previously reported as 1.33 and $-1.55 \mathrm{~cm}^{-1} / \mathrm{GPa}$ for tetragonal $\left(250 \mathrm{~cm}^{-1}\right)$ and monoclinic $\left(460 \mathrm{~cm}^{-1}\right)$ phase bands, respectively [17], $\Delta v$ can be considered to be a direct measure of the residual stress trace stored within the volume probed by the laser beam for each spectral acquisition. In addition, the equilibrium stress $\left(\sigma_{\mathrm{eq}}\right)$ for 3Y-TZP was calculated according to the following equation [17]:

$$
\sigma_{\mathrm{eq}}=\left(1-V_{m}\right) \sigma_{t}+V_{m} \sigma_{m}
$$

where $\sigma_{t}$ and $\sigma_{m}$ represent the residual stress traces stored in the tetragonal and monoclinic phases, respectively. In the present study, the Raman peak positions obtained from the unused surfaces of Head (I) were taken as stress-free references in calculating the $\sigma$ values according to (2)-(3). Therefore, the obtained stress values represent the relative changes of residual stress with respect to the unused implant device.

Moreover, the thickness of the transformed zone, $T_{m}$, of Head (I) was determined through mathematical deconvolution, which is a procedure to clean up the experimental depth profile of $V_{m}$ from the averaging effect of the Raman probe upon solving the following equation (for simplicity, we omit the full details of derivation of the formula and numerical methodologies here, since they have been explicitly given elsewhere [18]):

$$
\begin{aligned}
& V_{m}\left(z_{0}\right) \\
& =\frac{\int_{z=0}^{T_{m}} V_{\max } \times \exp \left[-\left(z-z_{0}\right)^{2} / 2 R^{2}\right] d z+\int_{T_{m}}^{+\infty} V_{\max } \times\left(\exp \left[-V_{1}\left(z-T_{m}\right)\right]+V_{2}\right) /\left(1+V_{2}\right) \times \exp \left[-\left(z-z_{0}\right)^{2} / 2 R^{2}\right] d z}{\int_{z=0}^{T_{m}} \exp \left[-\left(z-z_{0}\right)^{2} / 2 R^{2}\right] d z+\int_{T_{m}}^{+\infty} \exp \left[-\left(z-z_{0}\right)^{2} / 2 R^{2}\right] d z},
\end{aligned}
$$

where $z_{0}$ and $z$ are the focal position in the head surface and subsurface, respectively; $V_{\max }$ is the true maximum value of $V_{m} ; R$ is the radius of the waist of the laser beam; and $V_{1}, V_{2}$, and $T_{m}$ are the fitting parameters. The least-square curve fitting of the experimental data according to (4) was performed with the aid of commercially available software (Mathematica 7; Wolfram Research, IL).

\section{Results and Discussion}

3.1. Environmental Stability and LTD Kinetics of the 3Y-TZP Femoral Heads. Figures 1(a)-1(b) show representative Raman spectra collected from Head (I) before and after a $20 \mathrm{~h}$ aging period at $134^{\circ} \mathrm{C}$. In the as-received state of Head (I), the observed Raman bands indicate predominant spectral 


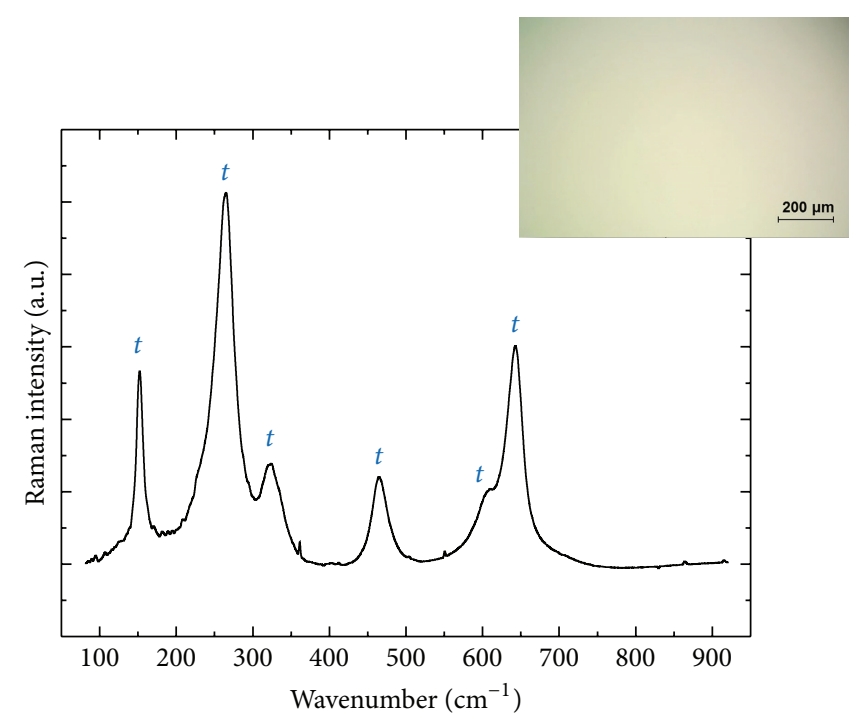

(a) Before aging



(b) After $20 \mathrm{~h}$ aging at $134^{\circ} \mathrm{C}$

FIgURE 1: Average Raman spectra and optical microscopic images collected at the surfaces of Head (I) before (a) and after $20 \mathrm{~h}$ aging at $134^{\circ} \mathrm{C}$ (b).



FIGURE 2: Raman spectroscopic data for the Head (I) surfaces showing the evolution of $t \rightarrow m$ phase transformation as a function of hydrothermal aging time in vitro.

contributions from tetragonal phase, while the bands from the monoclinic phase become the most preponderant after $20 \mathrm{~h}$ exposure to water vapor. Representative optical micrographs of the Head (I) surface are also given in the upperright of each figure. Figure 1(b) reveals the homogeneous formation of transformation-induced surface roughness with significant uplifts after autoclaving. The mean volume fractions of transformed 3Y-TZP $\left(V_{m}\right)$ were calculated from the fitted spectra of Head (I) surfaces using (1) and plotted as a function of in vitro aging time in Figure 2. The presence of a small fraction of monoclinic phase $\left(V_{m}=4.9 \%\right)$ was detected by Raman spectroscopy on the unused surface of Head (I), which was formed during the manufacturing process. The moisture-induced $t \rightarrow m$ transformation occurred slowly up to 5 hours aging, but it proceeded rapidly after 7.5 hours. This behavior can be interpreted according to a nucleation/growth model $[3,8,19-21]$. According to this model, the slow increase in monoclinic fraction observed at the initial stage of aging is due to the formation of monoclinic nuclei on the head surface. On the other hand, the sudden rise in monoclinic fraction after 7.5 hours aging represents the initiation of nuclei coalescence and, ultimately, the extension toward the bulk of the material.

In order to rationalize the LTD behavior of Head (I), the Mehl-Avrami-Johnson (MAJ) theory was applied. It has been previously reported that the kinetics of $t \rightarrow m$ transformation induced by aging can be expressed according to a modified MAJ equation $[3,8,19-23]$ :

$$
V_{m}=1-\left(1-V_{m}^{0}\right) \exp \left[-(b t)^{n}\right],
$$

where $V_{m}{ }^{0}$ is the pristine monoclinic phase fraction in the material before the aging test, $b$ is a parameter that represents the temperature dependence of the aging effect, $t$ is the aging duration, and $n$ is the time exponent, independent of temperature (generally referred to as the Avrami exponent). It should be noted that the $n$ value reflects the proportion of nucleation and growth rates. Previous experimental data and simulations showed that the $n$ exponent ranged between 0.5 and $4[3,20]$. An $n$ value close to 4 represents a preponderant contribution to the kinetics of transformation from the growth of preexisting monoclinic nuclei, while the nucleation rate is dominant at small $n$ value $[3,8,19-23]$. The $n$ exponent can be derived from the logarithmic form of (5) as follows:

$$
\ln \left(\ln \left(\frac{1-V_{m}^{0}}{1-V_{m}}\right)\right)=n \ln (b)+n \ln (t) .
$$

On the basis of the present experimental data, the obtained exponent value was $n=1.19\left(R^{2}=0.9835\right)$ as calculated 


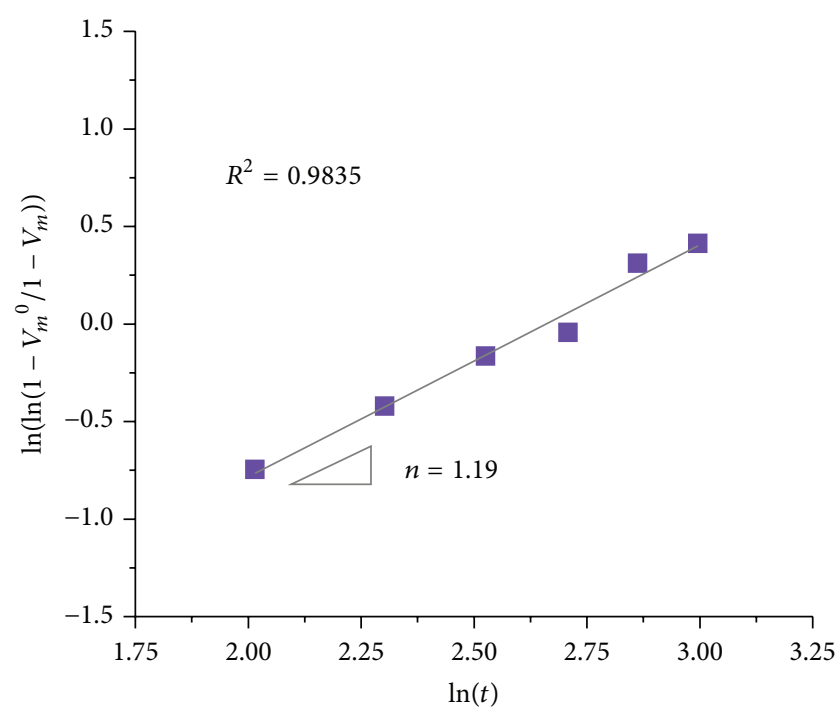

Figure 3: Plot of $\ln \left(\ln \left(1-V_{m}{ }^{0} / 1-V_{m}\right)\right)$ versus $\ln (t)$ for the determination of the Avrami exponent, $n$, for polymorphic transformation in Head (I). The exponent was obtained as $n=1.19$ by retrieving the slope of the best regression line for the plot obtained by Raman spectroscopic measurement.

from the slope of the best regression line to a plot $\ln (\ln (1-$ $\left.V_{m}{ }^{0} / 1-V_{m}\right)$ ) versus $\ln (t)$ (Figure 3 ). The $n$ value between 1 and 2 corresponds to a nucleation and one-dimensional growth process [21]. A similar evaluation based on MAJ theory was made by other authors for a commercial femoral head made of zirconia-toughened alumina matrix composite (ZTA) with the trade name of BIOLOX delta (CeramTec AG, Plochingen, Germany), which is presently one of the most advanced ceramic femoral heads. The $n$ values of BIOLOX delta were previously reported as $0.60 \sim 0.78[8,22,23]$. The studied Head (I) had a higher Avrami exponent as compared to BIOLOX delta, suggesting a faster growth rate of monoclinic nuclei in the LTD process. In other words, Prozyr head is expected to present faster kinetics of LTD during implantation.

\subsection{Effects of Transformation-Induced Stresses on the 3Y-TZP} Femoral Heads. Figures 4(a)-4(b) show the time-dependent plots of the average residual stresses stored in the tetragonal and monoclinic phases ( $\sigma_{t}$ and $\sigma_{m}$, resp.) in Head (I) surfaces. As shown in these plots, polymorphic transformation generated tensile and compressive stresses in the tetragonal and monoclinic grains of Head (I), respectively. Residual stress accumulation clearly proceeded with increasing aging time. According to stress assessments made by Schubert and Frey [24], the maximum tensile stresses induced by destabilization of the tetragonal polymorph were approximately evaluated as $300 \sim 500 \mathrm{MPa}$, which is a stress range similar to that detected in this study. The stress magnitudes rapidly increased at 7.5 hours aging presumably due to initiation of the growth process for monoclinic nuclei. The monoclinic phase incorporated strong residual compressive stresses in the GPa order in Head (I) surfaces, whose values reached a saturation plateau after 10 hours. The equilibrium residual stress, $\sigma_{\text {eq }}$, is also plotted in Figure 4(c), according to (3). Influenced by the quite strong compression generated in the monoclinic phase, the $\sigma_{\text {eq }}$ value at the head surface increased toward the compression side with increasing aging time. However, extremely high stress gradients can be envisaged on the head surface, which mechanically destabilize the bearing surface. It should be noted that the LTD-induced tensile stresses are responsible for surface microcracking, and ultimately, for fracture of the prosthesis. Indeed, after 15 hours aging, crack formation and grain detachment were clearly observed by optical microscopy on the surfaces of Head (I) (cf. Figures 5(a)-5(b)), which incorporated a tensile stress $>300 \mathrm{MPa}$ in the tetragonal phase (cf. Figure 4(a)). The largest tensile stresses could be found at grain junctions and edges where nucleation of transformation initiated, thus leading to the formation of cracks predominantly along grain boundaries [3].

Crack and grain-detachment formation, as observed in Figure 5, allows moist vapor to penetrate toward the head subsurface. The moisture can flow through grain boundary cracks and pores much faster than diffusion of hydroxyl ions into the lattice of individual grains [3]. Monoclinic phase fractions were plotted in Figure 6(a) as a function of indepth distance along the subsurface in Head (I) after aging for increasing periods of time. All the analyzed samples exhibited the highest contents of phase transformation at their surface. These data prove that phase transformation actually proceeds from the free surface toward subsurface regions with increasing exposure time. The thickness of the transformed zone $\left(T_{m}\right)$ was plotted in Figure 6(b) as a function of the aging time, which was determined from the deconvoluted monoclinic profile, $V_{m}(z)$. According to the least-square method, the best-fitting curves determined using (4) with minimum deviations from the data points were also plotted in the inset of the figure, and the good agreement between theoretical and experimental plots was confirmed, indicating high degree of precision and reproducibility of the results. Figure 6(b) illustrates that the transformation process of Head (I) can propagate in a nearly linear way (slope = $\left.0.19 \mu \mathrm{m} / \mathrm{h}, R^{2}=0.9587\right)$, and the $T_{m}$ value was $3.8 \mu \mathrm{m}$ after the $20 \mathrm{~h}$ exposure in vapor atmosphere. A simple linear time dependency in the aging kinetics of 3Y-TZP materials was also reported in the other recent studies [18, 25-28].

Since the tetragonal phase at the surface of the sample is more exposed (and unstable) as compared to the subsurface, the environmental stability is strongly dependent on the surrounding grains. Note that the gradual development of equilibrium residual stress is also observed within the first $50 \mu \mathrm{m}$ below the free surfaces due to the progress of polymorphic transformation (Figure 7).

3.3. Comparison of LTD Kinetics for In Vitro and In Vivo Environments. In the above Sections 3.1 and 3.2, the timedependent LTD behavior of monolithic 3Y-TZP was simulated according to in vitro hydrothermal aging tests and nondestructively characterized by Raman microprobe spectroscopy. According to the rationale behind both ISO and ASTM standards [8-11], the in vitro aging time should 


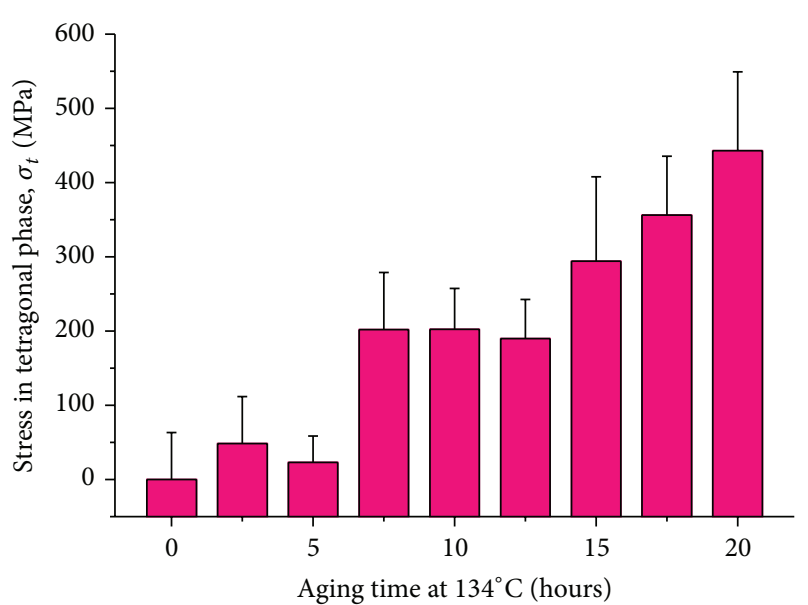

(a) Tetragonal phase

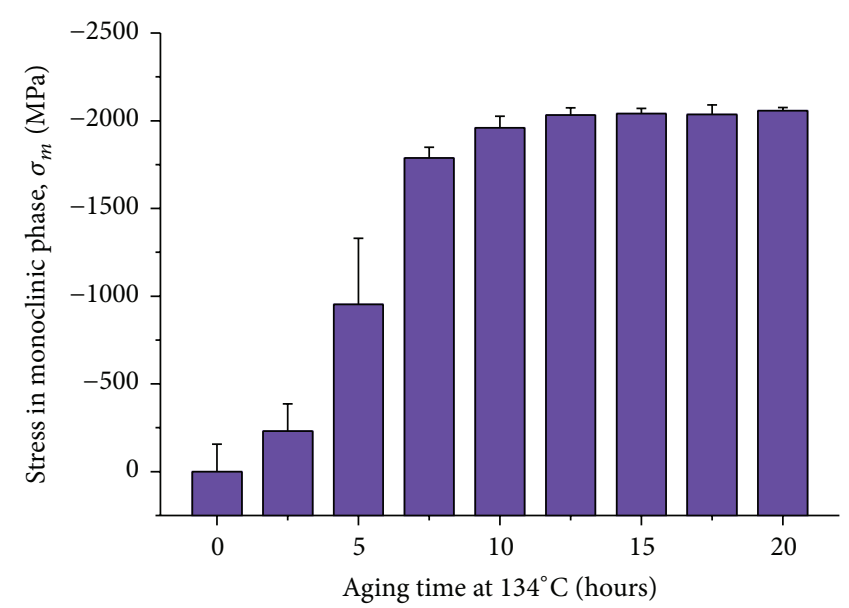

(b) Monoclinic phase

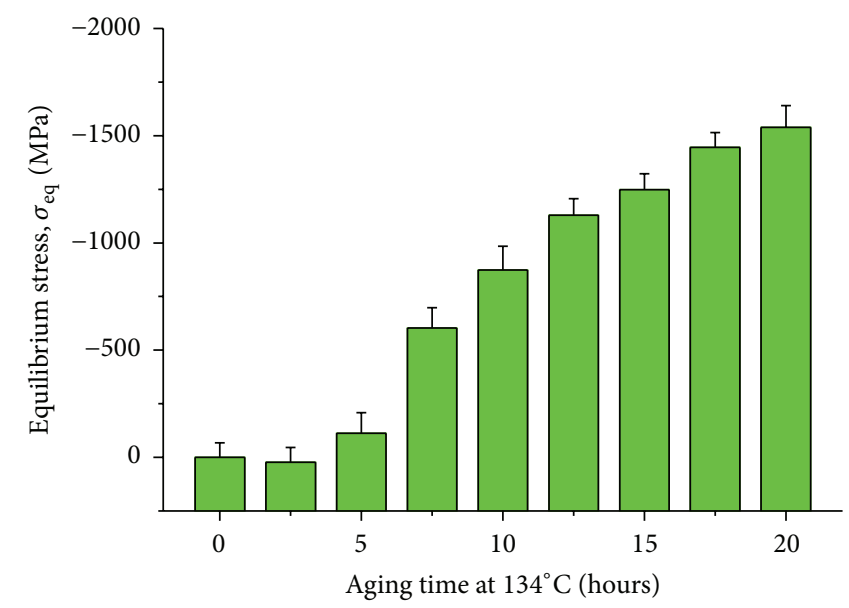

(c) Equilibrium

FIGURE 4: Raman spectroscopic data for the Head (I) surface showing the evolution of transformation-induced residual stresses in tetragonal (a) and monoclinic phase (b) as a function of the aging time. The equilibrium stress obtained according to (3) is also plotted in (c).

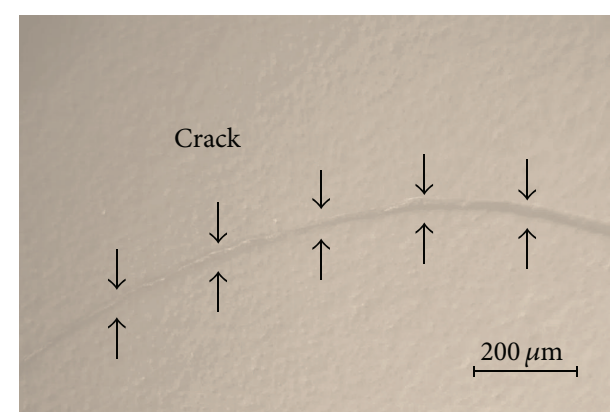

(a)

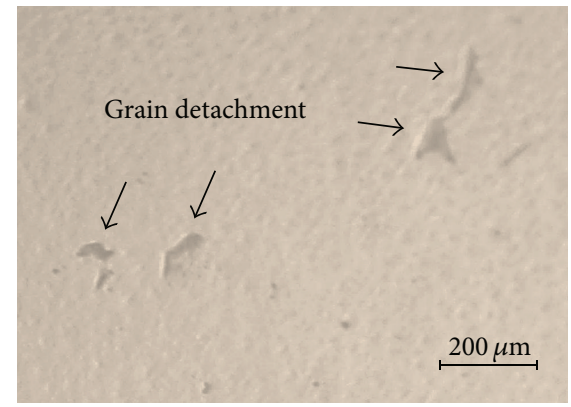

(b)

FIGURE 5: Optical micrographs showing surface crack propagation (a) and grain detachment (b) in Head (I) after 15 hours aging at $134^{\circ} \mathrm{C}$.

theoretically be comparable to in vivo implantation periods. Nevertheless, when converting from in vitro to in vivo times according to activation energy arguments, one can find a clear discrepancy between ISO and ASTM criteria. It was reported that, for medical grade 3Y-TZP, one-hour exposure at $134^{\circ} \mathrm{C}$ under 2 bars water steam corresponds approximately to 4 years in vivo exposure according to ISO standard $[8,9]$. On the other hand, ASTM F2345-03 defined that one-hour exposure at the above condition corresponds to 2 years in vivo $[10,11]$, which indeed represents a more severe condition for 3Y-TZP because of a shorter in vivo time for the same aging duration. The origin for this discrepancy lies in a difference 


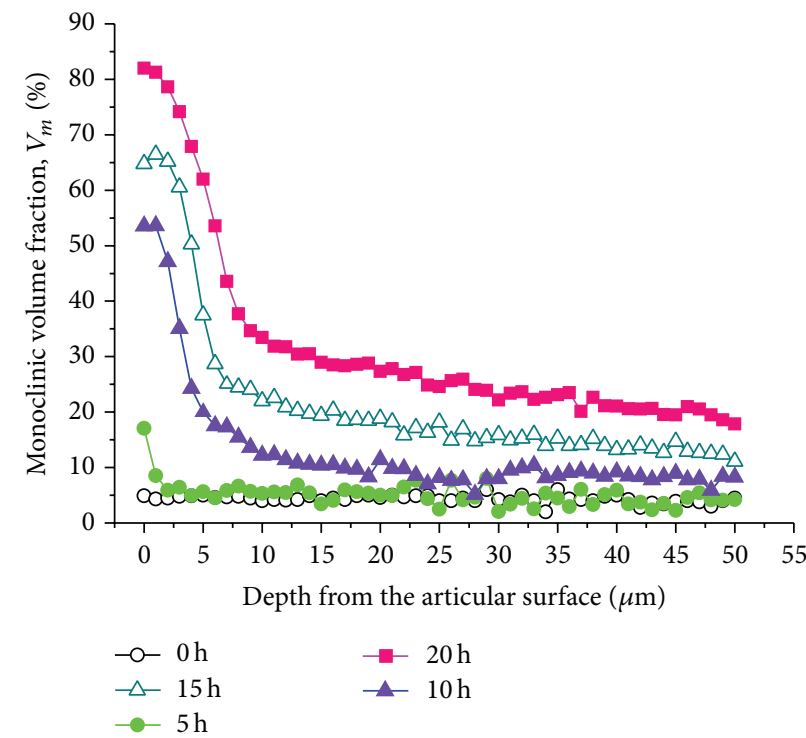

(a)

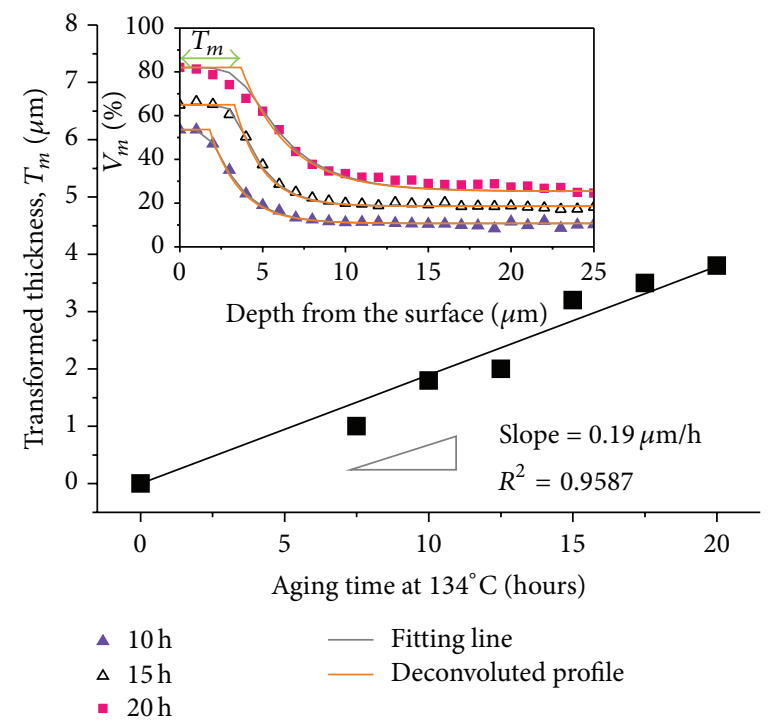

(b)

FIGURE 6: (a) Depth profiles of monoclinic phase fraction in Heads (I) after hydrothermal aging for different periods of time and (b) thickness of the transformed zone as a function of the aging time. The slope of the transformed thickness versus aging time indicates the rate of transformation propagation. The representative best-fitting curves of the experimental data (after 10, 15, and 20 hours aging) using (4) and deconvoluted monoclinic fraction profiles are also shown in the inset of (b).

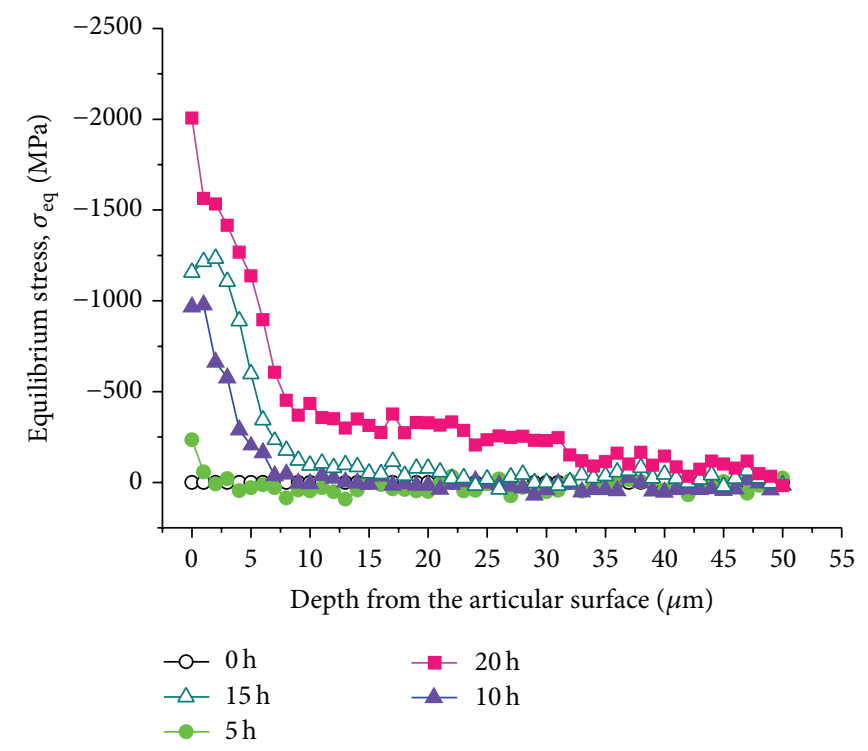

FIGURE 7: Depth profiles of equilibrium residual stress in Heads (I) after hydrothermal aging for different periods of time.

in the activation energy assumed for the extrapolation to in vivo temperature. In this context, in vitro aging criteria should not be considered as rigorous and universally true in lifetime extrapolation in vivo, but just as a rough indication. However, despite the fact that the most frequently published studies to date refer to ISO standard, we shall show hereafter that this standard is not the most appropriate for 3Y-TZP assessments.
In Figures 8(a) and 8(b), the monoclinic contents on the surface of Head (I) aged in vitro were compared with the $3 \mathrm{Y}$ TZP head retrievals (Heads (II) and (III)) and other values obtained from the literature; plots are given as a function of in vivo times simulated according to ISO and ASTM recommendations, respectively. These plots can be helpful in understanding how realistic these standards are, and this provides the most practical estimation for the biological environment. In addition to our long-term retrievals (15.1 and $16.6 \mathrm{y}$ ), we also introduced in the plot results of Prozyr retrievals after short- and middle-term implantation (1.6 $11 \mathrm{y})$, which had previously been reported in the literature [12-14]. Stewart et al. [12] reported 4.48 7.75\% monoclinic contents between 1.6 and 4 years follow-up in vivo, while Chevalier [13] reported 10\% transformation after 8 years in vivo. Hernigou and Bahrami [14] detected higher monoclinic contents at the levels of 19,25 , and $30 \%$ after 8,10 , and 11 years of in vivo implantation, respectively. It should be noted that the data plotted for Heads (II) and (III) represent the surface monoclinic fractions detected in their nonwear zones, predominantly including the influence of biological environment itself without any significant wear and load. According to Figure 7, the ASTM profile shows a much better agreement with the in vivo results as compared to the ISO profile. Accordingly, the ASTM recommendation appears as a more reasonable indication for predicting the rate of environmentally driven polymorphic transformation in medical grade $3 Y-T Z P$. The obtained results also imply that, in Prozyr 3Y-TZP femoral head components, nucleation of monoclinic sites should be expected approximately within the first 10 years of implantation. Subsequently, an exponential 


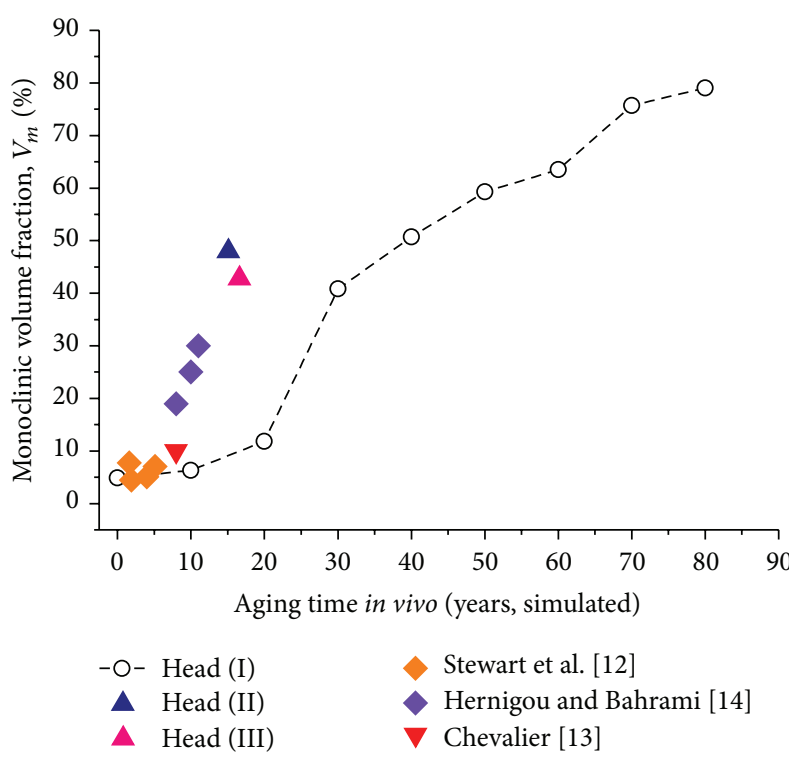

(a) ISO simulation

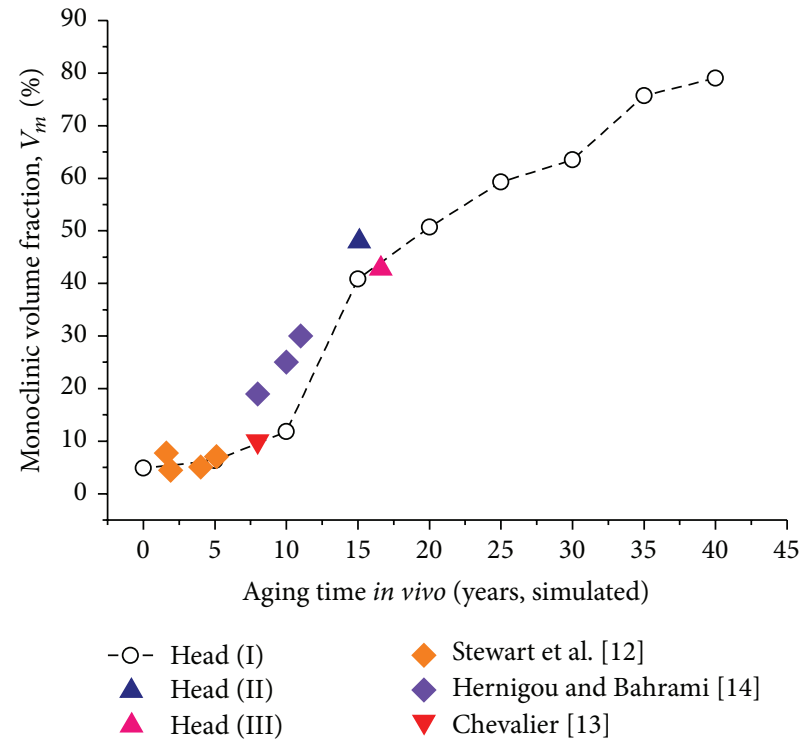

(b) ASTM simulation

FIGURE 8: Monoclinic phase fractions on the surface of Head (I) are plotted as a function of in-vivo time simulated according to ISO (a) and ASTM criteria (b). Note that the comparisons of the transformation kinetics are made between in-vitro and in-vivo environment by coplotting the transformed contents on the surfaces of Heads (II) and (III) as well as monoclinic contents reported for Prozyr femoral head retrievals in [12-14].

growth of monoclinic nuclei occurs, leading to a markedly enhanced risk of surface roughening and fracture.

Here, we would like to highlight several potential limitations of this study for the readers' convenience. The first one certainly was the limited sampling of retrieved Prozyr femoral heads after various implantation periods (only a total of ten retrieval data collected after short- to long-term services were considered here, according to the currently available literature data [12-14]). Secondly, the femoral heads used in this study were the first generation of monolithic 3Y-TZP introduced clinically in 1989. Thus, the obtained LTD behaviors are not generalizable to other $\mathrm{ZrO}_{2}$-containing prostheses with different constituents, grain sizes, and manufacturing processes. In addition, it should be noted that, as seen in Figure $8(\mathrm{~b})$, the middle-term retrievals (8 11 years) exhibited larger $V_{m}$ values as compared to the ASTM simulation line. Since the literature data were obtained from the wear zones of each retrieval surface [12-14], an additional contribution of biomechanical origin should be considered on top of the hydrothermal effect on polymorphic transformation. Indeed, the wear zones in the longer-term retrievals of Head (II)-(III) had significantly higher contents of monoclinic polymorphs (detected as 66.8 and $54.5 \%$, resp.) as compared to their respective nonwear zones. Thus, the interpretation of aging simulation for a long duration time should strictly be limited to predictions of the in vivo LTD in a nonwear zone. However, despite these limitations, we showed that the accelerated aging test could still be comparatively effective in investigating LTD processes and kinetics of $3 \mathrm{Y}-$ TZP femoral heads, giving reasonable predictions for the in vivo behavior, provided that the ASTM recommendation is adopted.

\section{Conflict of Interests}

All authors declare that there is no conflict of interests regarding the publication of this paper.

\section{Acknowledgment}

The authors sincerely thank Dr. W. Zhu for the many helpful advices and suggestions on the mathematical treatment.

\section{References}

[1] M. N. Rahaman, A. Yao, B. S. Bal, J. P. Garino, and M. D. Ries, "Ceramics for prosthetic hip and knee joint replacement," Journal of the American Ceramic Society, vol. 90, no. 7, pp. 19651988, 2007.

[2] I. C. Clarke, M. Manaka, D. D. Green et al., "Current status of zirconia used in total hip implants," The Journal of Bone and Joint Surgery-American Volume, vol. 85, no. 4, pp. 73-84, 2003.

[3] J. Chevalier, L. Gremillard, A. V. Virkar, and D. R. Clarke, "The tetragonal-monoclinic transformation in zirconia: lessons learned and future trends," Journal of the American Ceramic Society, vol. 92, no. 9, pp. 1901-1920, 2009.

[4] J. J. Jacobs, J. L. Gilbert, and R. M. Urban, "Corrosion of metal orthopaedic implants," The Journal of Bone and Joint SurgeryAmerican Volume, vol. 80, no. 2, pp. 268-282, 1998.

[5] G. Pezzotti, K. Yamada, A. A. Porporati, M. Kuntz, and K. Yamamoto, "Fracture toughness analysis of advanced ceramic composite for hip prosthesis," Journal of the American Ceramic Society, vol. 92, no. 8, pp. 1817-1822, 2009.

[6] C. Piconi, G. Maccauro, L. Pilloni, W. Burger, F. Muratori, and H. G. Richter, "On the fracture of a zirconia ball head," Journal of 
Materials Science: Materials in Medicine, vol. 17, no. 3, pp. 289300, 2006.

[7] G. Pezzotti, T. Saito, Y. Takahashi, K. Fukatsu, and N. Sugano, "Surface topology of advanced alumina/zirconia composite femoral head as compared with commercial femoral heads made of monolithic zirconia," Journal of the American Ceramic Society, vol. 94, no. 3, pp. 945-950, 2011.

[8] J. Chevalier, S. Grandjean, M. Kuntz, and G. Pezzotti, "On the kinetics and impact of tetragonal to monoclinic transformation in an alumina/zirconia composite for arthroplasty applications," Biomaterials, vol. 30, no. 29, pp. 5279-5282, 2009.

[9] G. Pezzotti, T. Saito, G. Padeletti, P. Cossari, and K. Yamamoto, "Nano-scale topography of bearing surface in advanced alumina/zirconia hip joint before and after severe exposure in water vapor environment," Journal of Orthopaedic Research, vol. 28, no. 6, pp. 762-766, 2010.

[10] V. Corfield, I. Khan, and R. Scott, "Hydrothermal stability of ceramic femoral heads," in Bioceramics and Alternative Bearings in Joint Arthroplasty, J. D. Chang and K. Billau, Eds., pp. 59-64, Steinkopff, Dresden, Germany, 2007.

[11] ASTM International Designation: F2345-03, "Standard test methods for determination of static and cyclic fatigue strength of ceramic modular femoral heads," in Annual Book of ASTM Standards, pp. 1-8, ASTM International, West Conshohocken, $\mathrm{Pa}, \mathrm{USA}, 2004$.

[12] T. Stewart, N. Flemming, M. Wroblewski, and J. Fisher, "The stability and durability of zirconia femoral heads," in Proceedings of the 51st Annual Meeting of the Orthopaedic Research Society, Poster no. 1167, Washington, DC, USA, February 2005.

[13] J. Chevalier, "What future for zirconia as a biomaterial?" Biomaterials, vol. 27, no. 4, pp. 535-543, 2006.

[14] P. Hernigou and T. Bahrami, "Zirconia and alumina ceramics in comparison with stainless-steel heads: polyethylene wear after a minimum ten-year follow-up," The Journal of Bone \& Joint Surgery-British Volume, vol. 85, no. 4, pp. 504-509, 2003.

[15] G. Katagiri, H. Ishida, A. Ishitani, and T. Masaki, "Direct determination by a Raman microprobe of transformation zone size in $\mathrm{Y}_{2} \mathrm{O}_{3}$-containing tetragonal $\mathrm{ZrO}_{2}$ polycrystals," in Advances in Ceramics, S. Somiya, N. Yamamoto, and H. Yanagida, Eds., pp. 537-544, American Ceramic Society, Westerville, Ohio, USA, 1988.

[16] G. Pezzotti, "Stress microscopy and confocal Raman imaging of load-bearing surfaces in artificial hip joints," Expert Review of Medical Devices, vol. 4, no. 2, pp. 165-189, 2007.

[17] M. Nawa, K. Yamada, and N. Kurizoe, "Effect of the t-m transformation morphology and stress distribution around the crack path on the measured toughness of zirconia ceramics: a case study on Ce-TZP/alumina nanocomposite," Journal of the European Ceramic Society, vol. 33, no. 3, pp. 521-529, 2013.

[18] W. Zhu, N. Sugano, and G. Pezzotti, "Nondestructive inspection of phase transformation in zirconia-containing hip joints by confocal Raman spectroscopy," Journal of Biomedical Optics, vol. 18, no. 12, Article ID 127002, 2013.

[19] W. A. Johnson and R. F. Mehl, "Reaction kinetics in processes of nucleation and growth," Transactions of the American Institute of Mining, Metallurgical and Petroleum Engineers, vol. 41, no. 11, pp. 2711-2775, 1939.

[20] L. Gremillard, J. Chevalier, T. Epicier, S. Deville, and G. Fantozzi, "Modeling the aging kinetics of zirconia ceramics," Journal of the European Ceramic Society, vol. 24, no. 13, pp. 3483-3489, 2004.
[21] Y. Takigawa, T. Shibano, Y. Kanzawa, and K. Higashi, "Effect of small amount of insoluble dopant on tetragonal to monoclinic phase transformation in tetragonal zirconia polycrystal," Materials Transactions, vol. 50, no. 5, pp. 1091-1095, 2009.

[22] G. Pezzotti, K. Yamada, S. Sakakura, and R. P. Pitto, "Raman spectroscopic analysis of advanced ceramic composite for hip prosthesis," Journal of the American Ceramic Society, vol. 91, no. 4, pp. 1199-1206, 2008.

[23] P. Taddei, E. Modena, F. Traina, and S. Affatato, "Raman and fluorescence investigations on retrieved Biolox delta femoral heads," Journal of Raman Spectroscopy, vol. 43, no. 12, pp. 18681876, 2012.

[24] H. Schubert and F. Frey, "Stability of Y-TZP during hydrothermal treatment: neutron experiments and stability considerations," Journal of the European Ceramic Society, vol. 25, no. 9, pp. 1597-1602, 2005.

[25] F. Zhang, M. Inokoshi, K. Vanmeensel, B. Van Meerbeek, I. Naert, and J. Vleugels, "Lifetime estimation of zirconia ceramics by linear ageing kinetics," Acta Materialia, vol. 92, pp. 290-298, 2015.

[26] M. Keuper, K. Eder, C. Berthold, and K. G. Nickel, "Direct evidence for continuous linear kinetics in the low-temperature degradation of Y-TZP," Acta Biomaterialia, vol. 9, no. 1, pp. 4826-4835, 2013.

[27] M. Dehestani and E. Adolfsson, "Phase stability and mechanical properties of zirconia and zirconia composites," International Journal of Applied Ceramic Technology, vol. 10, no. 1, pp. 129$141,2013$.

[28] J. Chevalier, J. Loh, L. Gremillard, S. Meille, and E. Adolfson, "Low-temperature degradation in zirconia with a porous surface," Acta Biomaterialia, vol. 7, no. 7, pp. 2986-2993, 2011. 


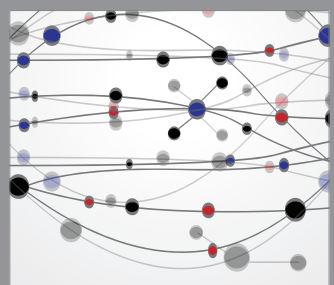

The Scientific World Journal
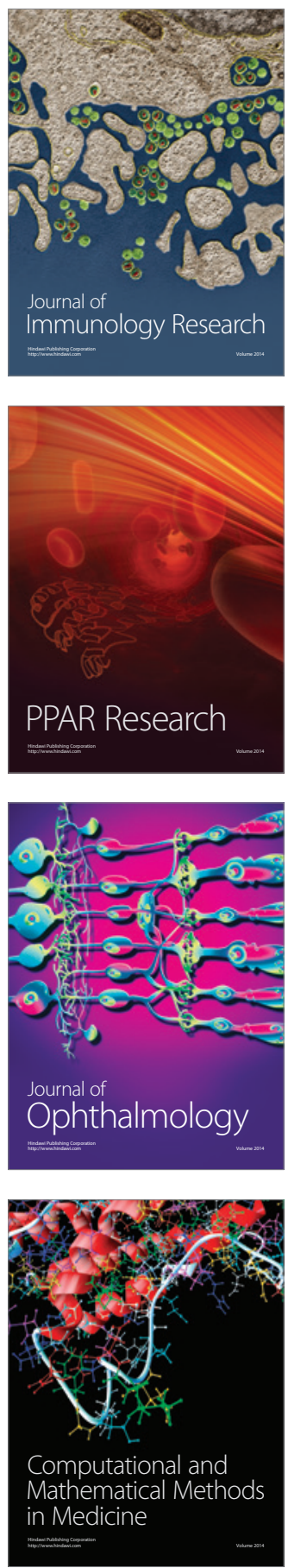

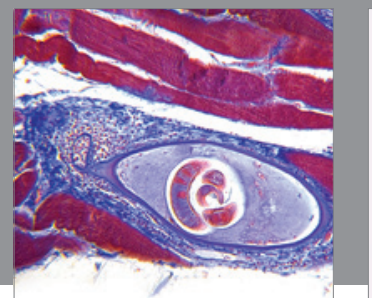

Gastroenterology

Research and Practice
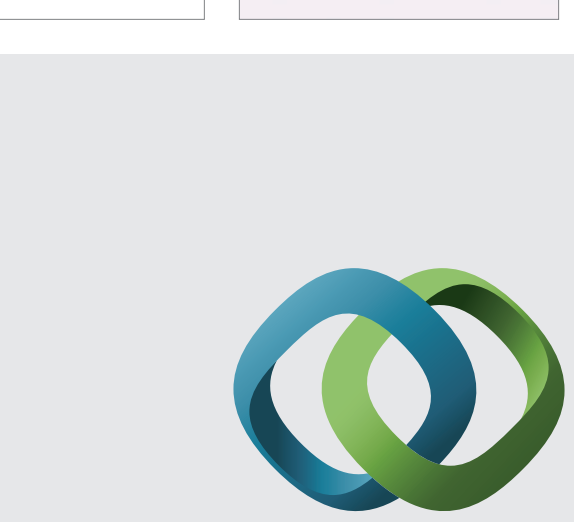

\section{Hindawi}

Submit your manuscripts at

http://www.hindawi.com
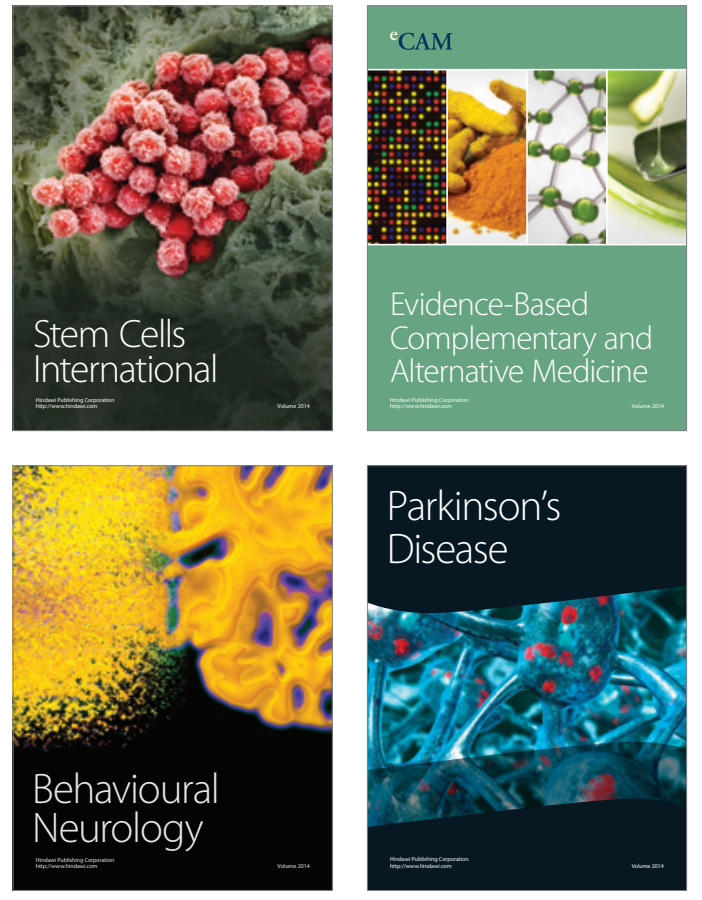
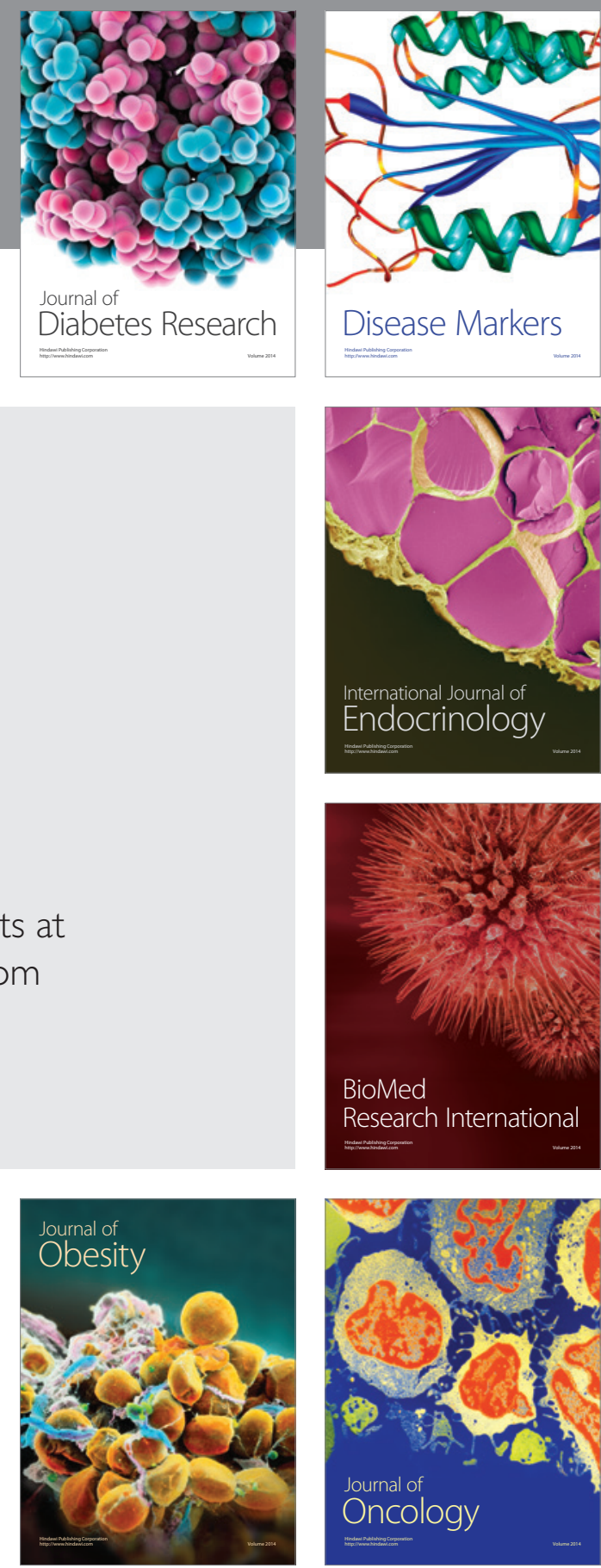

Disease Markers
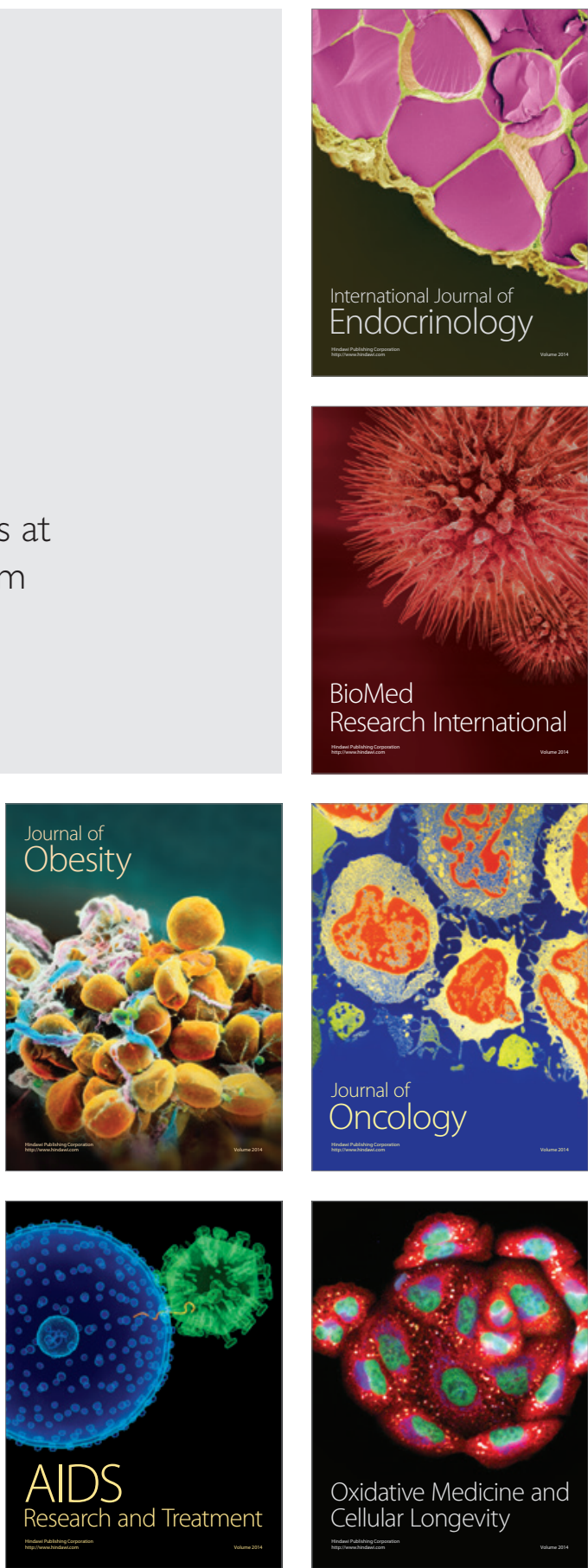